\title{
A Case Report of an Isolated Pulmonary Arteriovenous Malformation Causing Stroke
}

\author{
Mohammed Alhazzaa, Mukul Sharma, Grant Stotts
}

Can. J. Neurol. Sci. 2011; 38: 158-160

Up to $40 \%$ of strokes are cryptogenic ${ }^{1}$. This is particularly true in younger individuals. Occult cardiac sources of embolization, such as patent foramen ovale (PFO), have been postulated to explain this pattern ${ }^{2}$. A PFO is often implicated in young individuals with a suspected cardioembolic event. However, pulmonary arteriovenous malformations (PAVMs) can act as a right to left shunt and cause embolization bypassing the heart. Pulmonary arterio-venous malformations are commonly seen in patients with hereditary hemorrhagic telangiectasia (HHT), also known as Rendu-Osler-Weber disease. Here we present a case of a stroke related to an isolated pulmonary arteriovenous malformation (without HHT).

\section{CASE}

A 43-year-old, right-handed woman reported a transient episode of dysarthria and numbness of her left side during sexual intercourse. The symptoms gradually resolved over 60 minutes. She had a history of migraine with visual aura and Reynaud's disease with no other features suggestive of scleroderma. There was no history of atherosclerotic risk factors such as smoking, diabetes or hyperlipidemia. She had no features or family history of HHT. She was on low dose estrogen oral contraceptive pills. Her neurological, cardiac and respiratory examinations were normal.

A magnetic resonance image (MRI) demonstrated a remote left cortical infarction with high Fluid Attenuation Inversion Recovery (FLAIR) signal in the left middle frontal gyrus (Figure 1). Time of flight MR angiography of the head, neck and aortic arch was normal. Transesophegeal echocardiography revealed a significant opacification of the left chamber which appeared to occur late after contrast injection. Bubbles were not seen crossing the atrial septum, suggesting an extracardiac shunt. A computed tomogram (CT) pulmonary angiogram showed a tangle of abnormal vessels, located in the basal segment of the left lower lobe consistent with a large pulmonary arteriovenous malformation (Figure 2). There was no thrombus within the PAVM or evidence of pulmonary embolism on the CT pulmonary angiography. She had a normal bilateral lower extremity venous sonogram. Pelvic imaging was not obtained. Markers for thrombophilia were absent (including lupus anticoagulants, anticardiolipin antibodies, antithrombin, Factor V Leiden, Prothrombin gene mutation and Protein S and C levels). Due to the involvement of both hemispheres in this young woman, resulting in the MRI findings and the presenting transient ischemic attack (TIA), a central source of embolization was suspected and closure of the shunt was advised. Obliteration

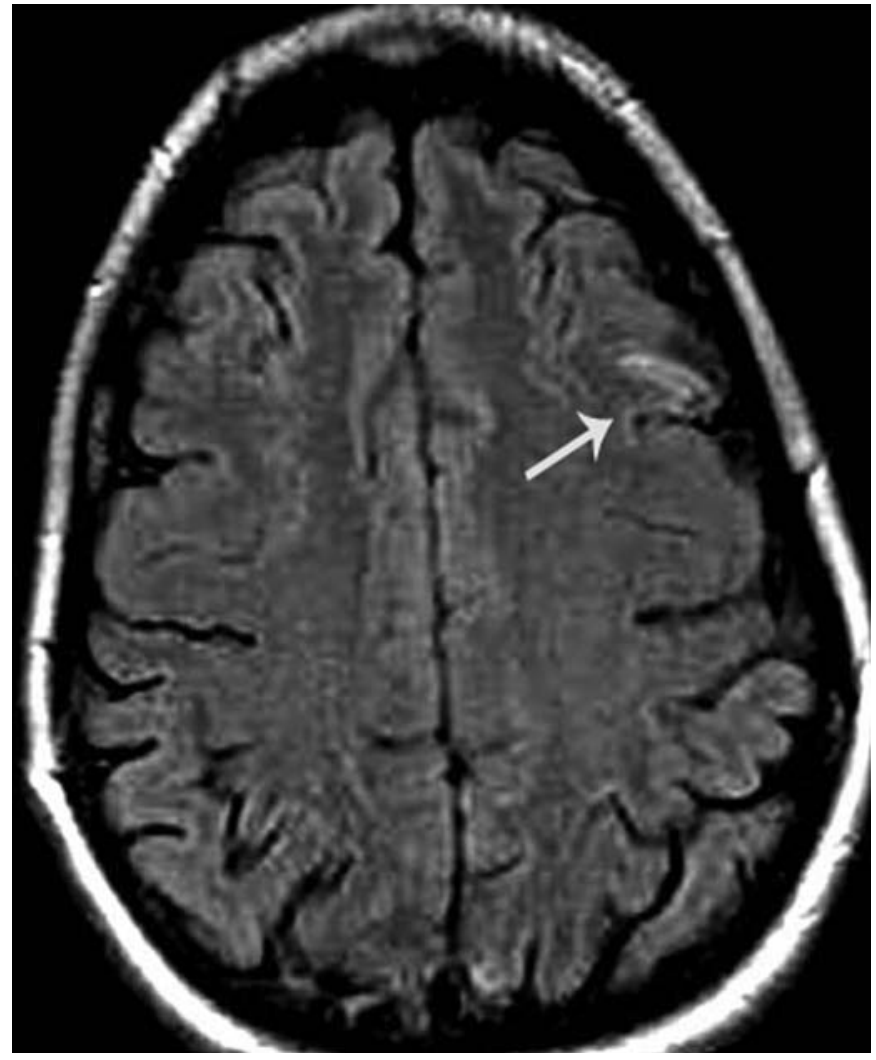

Figure 1: Brain MRI Fluid Attenuation Inversion Recovery (FLAIR) showing small left frontal cortical infarct.

of flow through the PAVM was achieved by embolization using coils. A CT pulmonary angiogram was obtained six months later and it showed sustained successful embolization. There have been no recurrent symptoms and, over 24 months, she has not reported any migraine attacks post-procedure.

From the Division of Neurology, Department of Medicine, The Ottawa Hospital, Ottawa, Ontario, Canada.

Received May 20, 2010. Final Revisions Submitted June 29, 2010.

Correspondence to: Grant Stotts, Department of Neurology, The Ottawa Hospital Civic Campus, C2, 1053 Carling Avenue, Ottawa, Ontario, K1Y 4E9, Canada. 


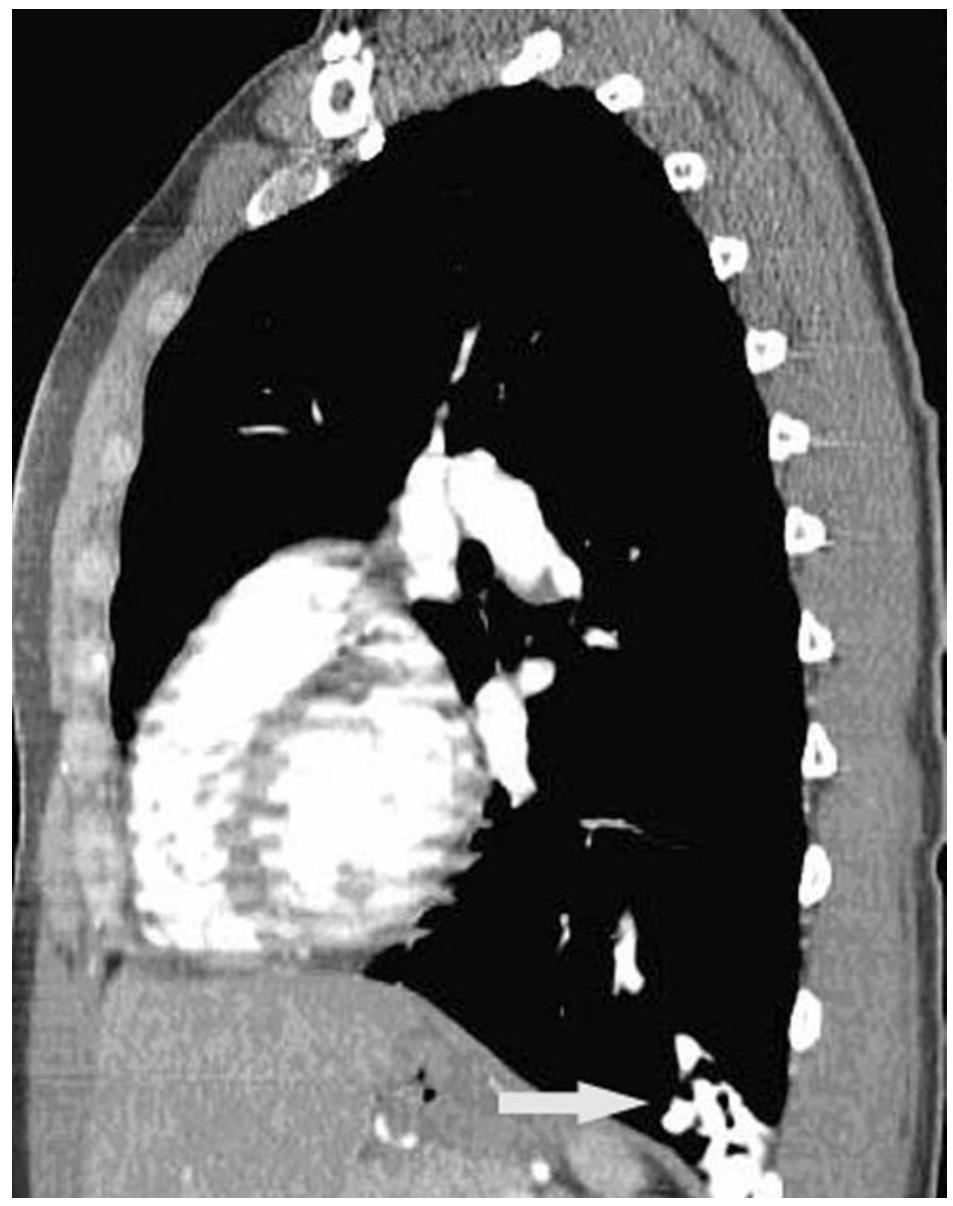

Figure 2: CT pulmonary angiogram showing left basal lower lobe pulmonary arteriovenous malformation (PAVM).

\section{Discussion}

Pulmonary arteriovenous malformations are abnormally dilated vessels with a thin-walled aneurysmal sac which provides a right- to-left shunt between the pulmonary arterial and venous circulations. Pulmonary arteriovenous malformations are commonly associated with HHT, an autosomal dominant disorder that results in vascular malformations in mucocutaneous tissues, visceral organs, and the central nervous system (CNS). Major clinical manifestations of HHT include dyspnea, epistaxis, gastro-intestinal hemorrhage and hepatic impairment ${ }^{3}$. Multiple cerebral lesions (such as strokes, brain abscess, or cerebral hemorrhage) have been recognized in 33\% to $50 \%$ of patients with $\mathrm{HHT}^{3,4}$. The prevalence of cerebral infarction (of all types) in a single PAVM is $32 \%$ and it increases to $60 \%$ in cases of multiple PAVMs ${ }^{4}$. Multiple PAVMs may be present with the risk of developing cerebral complications proportionate to the number of malformations ${ }^{4}$. Isolated PAVMs are relatively rare causes of stroke. In a series of 747 individuals with stroke only four were found to have an isolated PAVM ${ }^{5}$. Similar to PFO, the prevalence of migraine was found to be higher in patients with $\mathrm{PAVM}^{6}$. It is not clear whether the closure of PAVM would be beneficial for migraineurs. In our case, the patient had resolution of her migraines after closure. Further studies would be required to determine if embolization would be appropriate for migraineurs with PAVMs.

The proposed mechanism of cerebral involvement is through paradoxical embolization. Emboli may originate from the peripheral venous circulation (exogenous) or directly from a local thrombosis within the PAVM (endogenous) ${ }^{7}$. The risk may be further increased by polycythemia from PAVM induced hypoxia. Air embolism to the brain from a defect in the wall of the PAVM may also occur? ${ }^{7}$. In our case, investigations did not demonstrate evidence of endogenous or exogenous source. However, there is a high risk of thrombotic events in setting of migraine with aura and oral contraceptive pills use and these factors may play a role in this patient.

Pulmonary angiography remains the gold standard in the diagnosis of PAVMs. It can define the lesion number, location, size and identify malformations that may benefit from therapy. Transesophageal echocardiogram with bubble study may also aid in diagnosis. A finding of a right to left shunt during echocardiography should raise the possibility of PAVMs when 
there is a delayed contrast appearance in the left atrium with no atrial wall defects ${ }^{8}$. When there is an extracardiac shunt, the echocardiogram will show bubbles entering the left atrium three to eight cardiac cycles after they are seen in the right atrium (delayed opacification). In contrast, cardiac right to left shunts involve immediate opacification ${ }^{8}$. Chest X-ray may help as a screening tool; however, PAVMs were identified in only $45 \%$ of patients by this method in one series? .

Because of serious potential complications, treatment of PAVMs can be considered in asymptomatic patients ${ }^{10}$. Embolotherapy and surgical intervention are available options. Mager and colleagues evaluated the long-term results of embolotherapy and found successful long-term outcomes in $96 \%$ of patients in whom all angiographically visible PAVMs were treated ${ }^{10}$. They concluded that embolotherapy is relatively safe and efficacious ${ }^{10}$. Periprocedural complications occurred in $8 \%{ }^{10}$. Regular follow-up after embolotherapy is recommended since recanalization of occluded vessels or enlargement of untreated PAVMs can occur ${ }^{10}$. Surgical resection or ligation is rarely done now because of high morbidity and prolonged hospital stay. There are no prospective studies comparing surgical versus endovascular interventions.

In conclusion, early recognition of PAVMs is important given the significant potential for recurrent stroke and the existence of treatment options with low morbidity.

\section{REFERENCES}

1. Leys D, Bandu L, Henon H, et al. Clinical outcome in 287 consecutive young adults (15 to 45 years) with ischemic stroke. Neurology. 2002;59:26-33.

2. Lechat P, Mas JL, Lascault G, et al. Prevalence of patent foramen ovale in patients with stroke. New Eng J Med. 1988;318: 1148-52.

3. Cottin V, Chinet T, Lavole A, et al. Pulmonary arteriovenous malformations in hemorrhagic hereditary telangiectasia: a series of 126 patients. Medicine (Baltimore). 2007;86(1):1-17.

4. Moussouttas M, Fayad P, Rosenblatt M, et al. Pulmonary arteriovenous malformations: cerebral ischemia and neurologic manifestations. Neurology. 2000;55:959-64.

5. Kimura K, Minematsu K, Nakajima M, et al. Isolated pulmonary arteriovenous fistula without Rendu-Osler-Weber disease as a cause of cryptogenic stroke. J Neurol Neurosurg Psychiatry. 2004;75:311-13.

6. Thenganatt J, Schneiderman J, Hyland RH, et al. Migraines linked to intrapulmonary right-to-left shunt. Headache. 2006;46: 439-43.

7. Yeung M, Khan KA, Antecol DH, et al. Transcranial doppler ultrasonography and transesophageal echocardiography in the investigation of pulmonary arteriovenous malformation in a patient with hereditary hemorrhagic telangiectasia presenting with stroke. Stroke. 1995;26:1941-4.

8. Chessa M, Drago M, Krantunkov P, et al. Differential diagnosis between patent foramen ovale and pulmonary arteriovenous fistula in two patients with previous cryptogenic stroke caused by presumed paradoxical embolism. J Am Soc Echocardiogr. 2002; 15:845-6.

9. Kjeldsen AD, Oxhéj H, Andersen PE, et al. Prevalence of pulmonary arteriovenous malformations (PAVMs) and occurrence of neurological symptoms in patients with hereditary haemorrhagic telangiectasia (HHT). J Intern Med. 2000;248: 255-62.

10. Mager JJ, Overtoom TTC, Blauw H, et al. Embolotherapy of pulmonary arteriovenous malformations: long-term results in 112 patients. J Vasc Interv Radiol. 2004; 15:451-6. 